\title{
Allianz der Wissenschaftsorganisationen erhielt die Karl-Preusker-Medaille 2018
}

http://doi.org/10.1515/bd-2019-0013

Der Dachverband der Bibliotheksverbände, Bibliothek \& Information Deutschland (BID) e.V., verlieh die Karl-Preusker-Medaille 2018 an die Allianz der Wissenschaftsorganisationen. Die Bundesvereinigung würdigte damit das herausragende Engagement des Zusammenschlusses der bedeutendsten Wissenschaftsorganisationen Deutschlands zugunsten einer modernen Bibliotheksinfrastruktur. Die Auszeichnung wurde am 14. November 2018 im Grimm-Zentrum der Humboldt-Universität zu Berlin verliehen.

In ihrer Begründung hob die Jury hervor, dass die Allianz Bibliotheken seit genau zehn Jahren mit ihrer Schwerpunktinitiative „Digitale Information“ unterstützt und ihren Anliegen somit eine in der Politik weithin hörbare Stimme verleiht. Durch ihr Engagement im Bereich Open Access, Nationallizenzen und Forschungsprimärdaten fördert die Allianz Bibliotheken darin, den digitalen Wandel aktiv zu gestalten.

Mit der Verlängerung und Neuausrichtung der Schwerpunktinitiative „Digitale Information“ erklärt die Allianz ihre Bereitschaft, auch langfristig politische Grundsatzentscheidungen im Bereich der Digitalisierung zu koordinieren und weitere Ressourcen besonders für die Entwicklung des wissenschaftlichen Publikationssystems sowie für digitale Datensammlungen und Textkorpora bereit zu stellen.

Von besonderer Bedeutung für die nachhaltige Entwicklung der überregionalen Informationsversorgung ist das Engagement der Allianz beim Abschluss bundesweiter Lizenzverträge für das gesamte Portfolio elektronischer Zeitschriften großer Wissenschaftsverlage (Projekt DEAL).

Der Sprecher des Lenkungsausschusses des Projekts DEAL und Präsident der Hochschulrektorenkonferenz a. D., Prof. Dr. Dr. h. c. Horst Hippler, nahm die Karl-Preusker-Medaille am 14. November 2018 im Namen der Allianz im Jacobund-Wilhelm-Grimm-Zentrum der Humboldt-Universität zu Berlin entgegen. Die Laudatio hielt Prof. Dr. Ulrich Pöschl, Direktor am Max-Planck-Institut für Chemie in Mainz.

Die Karl-Preusker-Medaille erinnert an Karl Benjamin Preusker (1786-1871), der am 24. Oktober 1828 im sächsischen Großenhain eine Schulbibliothek gründete, aus der wenig später die erste Öffentliche Bibliothek in Deutschland hervorging. Die Medaille wird seit 1996 an Personen und Institutionen verliehen, die den Kultur- und Bildungsauftrag des Bibliothekswesens in herausragender 
Weise fördern und unterstützen. $\mathrm{Zu}$ den Persönlichkeiten, die bisher mit der Medaille ausgezeichnet wurden, gehören unter anderem Bundespräsident a. D. Horst Köhler, Ranga Yogeshwar und Paul Raabe. Weitere Informationen zur KarlPreusker-Medaille finden Sie im Internet unter www.bid.bideutschland.de/karlpreusker-medaille/.

\section{Kontakt:}

Bibliothek \& Information Deutschland (BID) e.V.

Dr. Monika Braß, BID-Geschäftsführerin,

Tel.: +49 (0)30/644989920

E-Mail: bid@bideutschland.de

Hochschulrektorenkonferenz (HRK)

Susanne Schilden, Pressesprecherin der Hochschulrektorenkonferenz (HRK)

Tel.: +49 (0)228 887-152

E-Mail: schilden@hrk.de 